\title{
Cognitive Artificial Intelligence Method for Interpreting Transformer Condition Based on Maintenance Data
}

Karel Octavianus Bachri, Bambang Anggoro, Arwin Datumaya Wahyudi Sumari, Adang Suwandi Ahmad*

Cognitive Artificial-Intelligence Research Group, School of Electrical Engineering and Informatics, Bandung Institute of Technology, Bandung, 40132, Indonesia

A R T I C L E I N F O

Article history:

Received: 06 April, 2017

Accepted: 24 June, 2017

Online: 16 July, 2017

Keywords:

Cognitive Artificial-Intelligence

A3S

OMA3S

Information Fusion

Transformer Condition

\begin{abstract}
A B S T R A C T
A3S(Arwin-Adang-Aciek-Sembiring) is a method of information fusion at a single observation and OMA3S(Observation Multi-time A3S) is a method of information fusion for time-series data. This paper proposes OMA3S-based Cognitive Artificial-Intelligence method for interpreting Transformer Condition, which is calculated based on maintenance data from Indonesia National Electric Company (PLN). First, the proposed method is tested using the previously published data, and then followed by implementation on maintenance data. Maintenance data are fused to obtain part condition, and part conditions are fused to obtain transformer condition. Result shows proposed method is valid for DGA fault identification with the average accuracy of $91.1 \%$. The proposed method not only can interpret the major fault, it can also identify the minor fault occurring along with the major fault, allowing early warning feature. Result also shows part conditions can be interpreted using information fusion on maintenance data, and the transformer condition can be interpreted using information fusion on part conditions. The future works on this research is to gather more data, to elaborate more factors to be fused, and to design a cognitive processor that can be used to implement this concept of intelligent instrumentation.
\end{abstract}

\section{Introduction}

In the earlier paper [1], transformer condition is used to estimate its end of life using instant data. In this paper, transformer condition is calculated using maintenance data from Indonesia Electric Company (PLN) and is interpreted using CognitiveArtificial method. Each factor influencing the same component is assumed to have the same weight in the condition calculation. The condition is then interpreted to provide early warning system for the potential failure and to estimate the transformer end of life.

There are no single conventional method of transformer diagnosis can be used to define transformer condition accurately. Usually there are several methods combined to perform such a task. These methods are very expert-dependant and are not formulated. Therefore, an automated method for transformer condition monitoring is proposed. Using Cognitive-Artificial-Intelligence (CAI) method, the transformer condition interpretation can be

\footnotetext{
${ }^{*}$ Corresponding Author: Adang Suwandi Ahmad, Cognitive Artificial-Intelligence Research Group, Bandung Institute of Technology, Bandung, Indonesia Email: adangsahmad@yahoo.com.
}

accurately performed, and the expert-dependency can be reduced as well.

\section{Transformer Condition Component}

Transformer condition can be calculated using some factors. These factors are shown in Figure 1 [2].

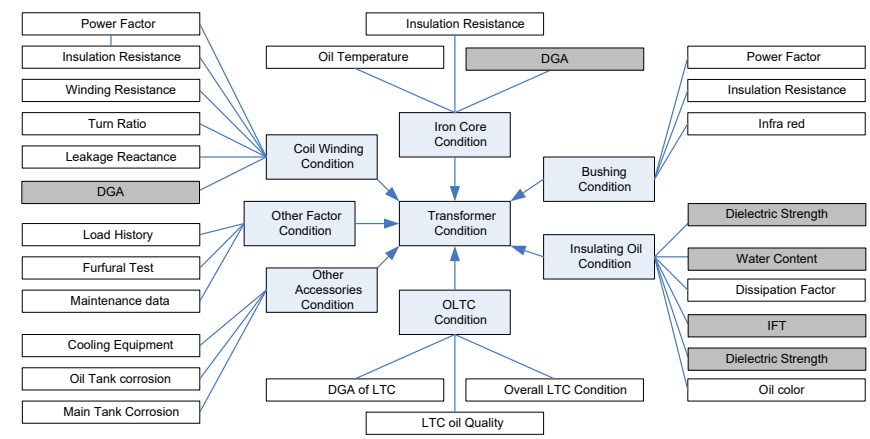

Figure 1 Transformer Condition Factors [2] 
Data are collected from PLN and fused to obtain part conditions, part conditions are then fused to obtain transformer condition. Figure 2 explains how transformers degrade over time.

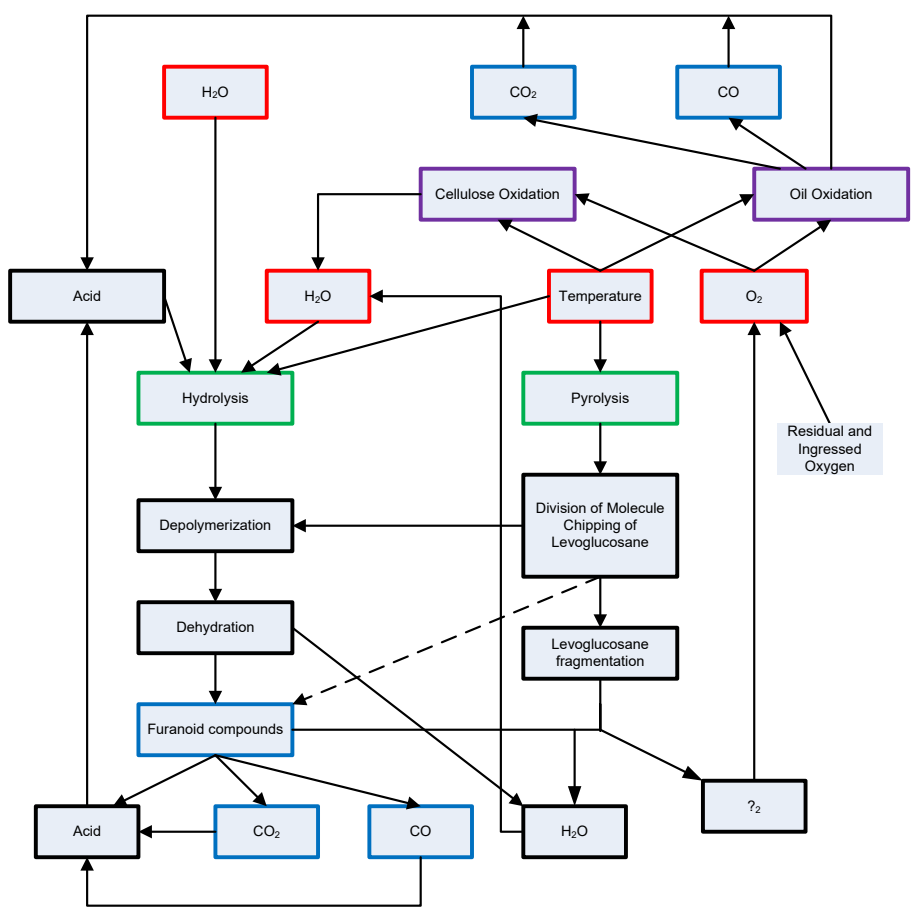

Figure 2 Transformer Degradation Diagram [3]

There are two main processes of the transformer degradation process, hydrolysis and pyrolysis. Hydrolysis is related to water, while pyrolysis is related to fire. The main cause of hydrolysis is water, acids and temperature causes hydrolysis as well. Pyrolysis is caused by temperature [3].

Hydrolysis causes depolymerization of transformer insulating system, and later produces furanoid compounds, which produces carbon dioxide and carbon monoxide, which is the main cause of acids [3]. Acid will then cause hydrolysis.

Pyrolysis causes levoglucosane fragmentation, which produces diatomic oxygen. Oxygen is the cause of oxidation in cellulose and oil, which leads to hydrolysis [3]. These two degradation processes and the compounds they produce makes the transformer degradation processes accelerate over time. The impact of diatomic oxygen will be discussed in Load Tap Changer.

\section{The Mathematical Model of Arwin-Adang-Aciek- Sembiring (A3S) [4]}

How knowledge grows in the system can be described using Figure $2[4,5]$. There are two main parts of Knowledge-Growing System. The upper part of Figure 3 contains Information Fusion, while the lower part of the diagram contains knowledge fusion.

The system receives multi-source information from sensors and performs information fusion. When the information exceeds certain level of desirable Degree of Certainty, the information will be considered as knowledge.
The knowledge will be fused with the existing knowledge in the knowledge part of the system to obtain new knowledge and is stored. When the new knowledge exceeds certain level of DoC, it will become the ultimate knowledge.

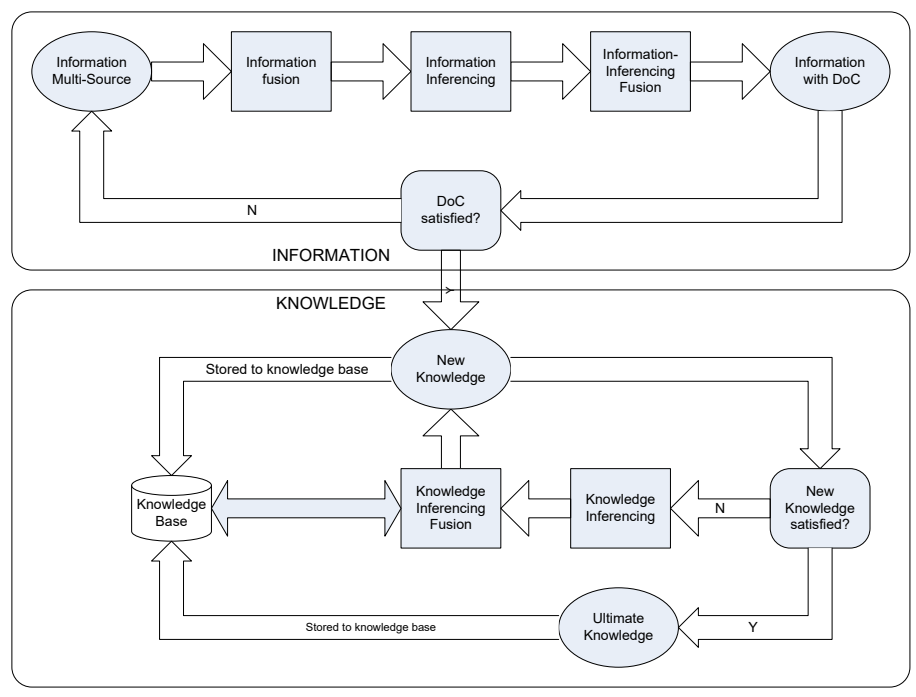

Figure 3 Knowledge-Growing System [5]

A3S (Arwin-Adang-Aciek-Sembiring) algorithm [5] is information fusion algorithm based on Bayesian Inference Method. When a problem occurs, the system collects information and fuses them to produce new knowledge. A3S starts with (1).

$$
P\left(B_{j} \mid A_{i}\right)=\sum_{i=1}^{n}\left(\frac{P\left(A_{i} \mid B_{j}\right) P\left(B_{j}\right)}{\sum_{k=1}^{m} P\left(A_{i} \mid B_{k}\right) P\left(B_{k}\right)}\right)
$$

Where $P\left(B_{j} \mid A_{i}\right)$ is the probability of $B_{j}$ is true given the presence of the fusion or combination of all events $A_{i}$ [4]. Maximum A Posteriori (MAP) is determined by (2)

$$
P\left(B_{j} \underset{\max }{+} A_{i}\right)_{\text {estimate }}=\max _{j}\left(\frac{P\left(B_{j} \mid A_{i}\right)}{n}\right)
$$

It is then simplified to become (3)

$$
P\left(\psi_{1}^{j}\right)=\frac{\sum_{i=1}^{\delta} P\left(v_{i}^{j}\right)}{\delta}
$$

Where $P\left(\psi_{i}^{j}\right)$ will be the New Knowledge Probability Distribution (NKPD) at a certain observation time $\gamma_{1}$ [4]. The new knowledge will be obtained by applying (4).

$$
P\left(\psi_{1}^{j}\right)_{\text {estimate }}=\max \left(\left[P\left(\psi_{1}^{j}\right)\right]\right)
$$


K. O. Bachri et al. / Advances in Science, Technology and Engineering Systems Journal Vol. 2, No. 3, 1137-1146 (2017)

The system will keep collecting information (NKPD) on each observation, $P\left(\psi_{1}^{j}\right), \ldots, P\left(\psi_{\gamma}^{j}\right), \ldots, P\left(\psi_{\Gamma}^{j}\right)$ [4]. The inferencing can be determined using (5).

$$
P\left(\phi_{\gamma}^{j}\right)= \begin{cases}1, & P\left(\psi_{\gamma}^{j}\right)>\frac{P\left(\psi_{\gamma}^{j}\right)}{\lambda} \\ 0, & P\left(\psi_{\gamma}^{j}\right) \leq \frac{P\left(\psi_{\gamma}^{j}\right)}{\lambda}\end{cases}
$$

Where $P\left(\phi_{\gamma}^{j}\right)$ is inferencing of each information to the knowledge distribution.

Information-inferencing fusion will be calculated using OMA3S method, a dynamic version of A3S resulting NKPD over Time (NKPDT) [4].

$$
\begin{gathered}
P\left(\theta_{j}\right)=\frac{\sum_{\chi=1}^{\Gamma} P\left(\phi_{\gamma}^{j}\right)}{\Gamma} \\
P(\theta)_{\text {estimate }}=\max \left\lfloor P\left(\theta_{j}\right)\right\rfloor
\end{gathered}
$$

\section{Transformer condition calculation}

System's block diagram is shown in Figure 4.

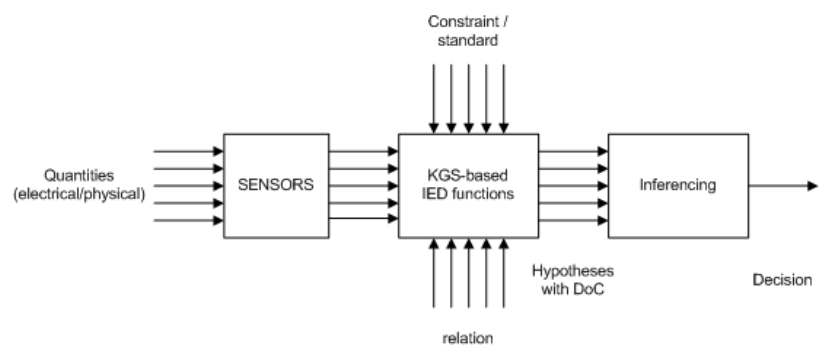

Figure 4 System's block diagram

Data is collected using sensors and is compared to standards and relation. The system fills the observation table using (1). After each observation, there will be new knowledge shown by New Knowledge Probability Distribution (NKPD). Each NKPD is fused with the previous NKPD to produce NKPD over time (NKPDT). Decisions are made based on NKPDT.

There are two kinds of faults in transformers. They are electrical faults and thermal faults [9]. Electrical faults are Partial Discharge, Low-Energy Discharge, and High Energy Discharge, while thermal faults are Thermal-Low and Thermal-High [9]. The proposed method is tested using previously published DGA dataset, which is classified based on the identified fault [9]. Table 1 shows Partial Discharge dataset, Table 2 shows Low-Energy Discharge, Table 3 shows High-Energy Discharge, Table 4 shows Thermal-Low, and Table 5 shows High-Energy Discharge.
Table 1 Partial Discharge dataset [9].

\begin{tabular}{|c|c|r|r|r|r|r|}
\hline No. & $\mathrm{H}_{2}$ & $\mathrm{CH}_{4}$ & $\mathrm{C}_{2} \mathrm{H}_{6}$ & $\mathrm{C}_{2} \mathrm{H}_{4}$ & $\mathrm{C}_{2} \mathrm{H}_{2}$ & $\mathrm{CO}$ \\
\hline 1 & 32930 & 2397 & 157 & 0 & 0 & 313 \\
\hline 2 & 37800 & 1740 & 249 & 8 & 8 & 56 \\
\hline 3 & 92600 & 10200 & 0 & 0 & 0 & 6400 \\
\hline 4 & 8266 & 1061 & 22 & 0 & 0 & 107 \\
\hline 5 & 9340 & 995 & 60 & 6 & 7 & 60 \\
\hline 6 & 36036 & 4704 & 554 & 5 & 10 & 6 \\
\hline 7 & 33046 & 619 & 58 & 2 & 0 & 51 \\
\hline 8 & 40280 & 1069 & 1060 & 1 & 1 & 1 \\
\hline 9 & 26788 & 18342 & 2111 & 27 & 0 & 704 \\
\hline
\end{tabular}

Table 2 Low-Energy Discharge dataset [9].

\begin{tabular}{|c|r|r|r|r|r|r|}
\hline No. & $\mathrm{H}_{2}$ & $\mathrm{CH}_{4}$ & $\mathrm{C}_{2} \mathrm{H}_{6}$ & $\mathrm{C}_{2} \mathrm{H}_{4}$ & $\mathrm{C}_{2} \mathrm{H}_{2}$ & $\mathrm{CO}$ \\
\hline 1 & 78 & 20 & 11 & 13 & 28 & 0 \\
\hline 2 & 305 & 100 & 33 & 161 & 541 & 440 \\
\hline 3 & 35 & 6 & 3 & 26 & 482 & 200 \\
\hline 4 & 543 & 120 & 41 & 411 & 1880 & 76 \\
\hline 5 & 1230 & 163 & 27 & 233 & 692 & 130 \\
\hline 6 & 645 & 86 & 13 & 110 & 317 & 74 \\
\hline 7 & 60 & 10 & 4 & 4 & 4 & 780 \\
\hline 8 & 95 & 10 & 0 & 11 & 39 & 122 \\
\hline 9 & 6870 & 1028 & 79 & 900 & 5500 & 29 \\
\hline
\end{tabular}

Table 3 High-Energy Discharge dataset [9].

\begin{tabular}{|c|r|r|r|r|r|r|}
\hline No. & $\mathrm{H}_{2}$ & $\mathrm{CH}_{4}$ & $\mathrm{C}_{2} \mathrm{H}_{6}$ & $\mathrm{C}_{2} \mathrm{H}_{4}$ & $\mathrm{C}_{2} \mathrm{H}_{2}$ & $\mathrm{CO}$ \\
\hline 1 & 440 & 89 & 19 & 304 & 757 & 299 \\
\hline 2 & 210 & 43 & 12 & 102 & 187 & 167 \\
\hline 3 & 2850 & 1115 & 138 & 1987 & 3675 & 2330 \\
\hline 4 & 7020 & 1850 & 0 & 2960 & 4410 & 2140 \\
\hline 5 & 545 & 130 & 16 & 153 & 239 & 660 \\
\hline 6 & 7150 & 1440 & 97 & 1210 & 1760 & 608 \\
\hline 7 & 620 & 325 & 38 & 181 & 244 & 1480 \\
\hline 8 & 120 & 31 & 0 & 66 & 94 & 48 \\
\hline 9 & 755 & 229 & 32 & 404 & 460 & 845 \\
\hline
\end{tabular}

Table 4 Thermal-Low dataset [9].

\begin{tabular}{|c|c|r|r|r|r|r|}
\hline No. & $\mathrm{H}_{2}$ & $\mathrm{CH}_{4}$ & $\mathrm{C}_{2} \mathrm{H}_{6}$ & $\mathrm{C}_{2} \mathrm{H}_{4}$ & $\mathrm{C}_{2} \mathrm{H}_{2}$ & $\mathrm{CO}$ \\
\hline 1 & 1270 & 3450 & 520 & 1390 & 8 & 483 \\
\hline 2 & 3420 & 7870 & 1500 & 6990 & 33 & 573 \\
\hline 3 & 360 & 610 & 259 & 260 & 9 & 12000 \\
\hline 4 & 1 & 27 & 49 & 4 & 1 & 53 \\
\hline 5 & 3675 & 6392 & 2500 & 7691 & 5 & 101 \\
\hline 6 & 48 & 610 & 29 & 10 & 0 & 1900 \\
\hline 7 & 12 & 18 & 4 & 4 & 0 & 559 \\
\hline 8 & 66 & 60 & 2 & 7 & 0 & 76 \\
\hline 9 & 1450 & 940 & 211 & 322 & 61 & 2420 \\
\hline
\end{tabular}


Table 5 Thermal-High dataset [9].

\begin{tabular}{|c|c|r|r|r|r|r|}
\hline No. & $\mathrm{H}_{2}$ & $\mathrm{CH}_{4}$ & $\mathrm{C}_{2} \mathrm{H}_{6}$ & $\mathrm{C}_{2} \mathrm{H}_{4}$ & $\mathrm{C}_{2} \mathrm{H}_{2}$ & $\mathrm{CO}$ \\
\hline 1 & 8800 & 64064 & 72128 & 95650 & 0 & 290 \\
\hline 2 & 6709 & 10500 & 1400 & 17700 & 750 & 290 \\
\hline 3 & 1100 & 1600 & 221 & 2010 & 26 & 0 \\
\hline 4 & 290 & 966 & 299 & 1810 & 57 & 72 \\
\hline 5 & 2500 & 10500 & 4790 & 13500 & 6 & 530 \\
\hline 6 & 1860 & 4980 & 0 & 10700 & 1600 & 158 \\
\hline 7 & 860 & 1670 & 30 & 2050 & 40 & 10 \\
\hline 8 & 150 & 22 & 9 & 60 & 11 & 0 \\
\hline 9 & 400 & 940 & 210 & 820 & 24 & 390 \\
\hline
\end{tabular}

In order to analyze the DGA, there are several ratios required, they are [5]:

$$
\begin{aligned}
& R_{1}=\left[\mathrm{CH}_{4}\right] /\left[\mathrm{H}_{2}\right] \\
& R_{2}=\left[\mathrm{C}_{2} \mathrm{H}_{2}\right] /\left[\mathrm{C}_{2} \mathrm{H}_{4}\right] \\
& R_{5}=\left[\mathrm{C}_{2} \mathrm{H}_{4}\right] /\left[\mathrm{C}_{2} \mathrm{H}_{6}\right]
\end{aligned}
$$

These ratios are put into groups based on Table 6 .

Table 6 Gas Ratio grouping [9].

\begin{tabular}{|c|c|c|c|}
\hline & R2 & R1 & R5 \\
\hline$<0.1$ & 0 & 1 & 0 \\
\hline $0.1-1.0$ & 1 & 0 & 0 \\
\hline $1.0-3.0$ & 1 & 2 & 1 \\
\hline$>3$ & 2 & 2 & 2 \\
\hline
\end{tabular}

Estimated faults can be determined using the rules shown in Table 7 [9].

Table 7 Gas Ratio grouping [9].

\begin{tabular}{|c|l|c|c|c|}
\hline No. & Characteristic Fault & R2 & R1 & R5 \\
\hline 0 & No fault & 0 & 0 & 0 \\
\hline 1 & Partial Discharge & 0 or 1 & 1 & 0 \\
\hline 2 & Low-Energy Discharge & 1 or 2 & 0 & 1 or 2 \\
\hline 3 & High-Energy Discharge & 1 & 0 & 2 \\
\hline 4 & Thermal-Low & 0 & 0 or 2 & 0 or 1 \\
\hline 5 & Thermal-High & 0 & 2 & 2 \\
\hline
\end{tabular}

Datasets are made into Ratios and are put into groups as shown in Table 8 to Table 12 .

Table 8 Ratios: Partial Discharge.

\begin{tabular}{|c|c|c|c|c|c|}
\hline \multicolumn{3}{|c|}{ Ratio } & \multicolumn{3}{|c|}{ Ratio Group } \\
\hline R1 & R2 & R5 & R1 & R2 & R5 \\
\hline 2.72 & 0.01 & 2.67 & 1 & 2 & 0 \\
\hline 2.30 & 0.00 & 4.66 & 1 & 1 & 0 \\
\hline 1.69 & 0.03 & 1.00 & 0 & 2 & 2 \\
\hline 27.00 & 0.25 & 0.08 & 0 & 2 & 0 \\
\hline 1.74 & 0.00 & 3.08 & 0 & 1 & 0 \\
\hline 12.71 & 0.00 & 0.34 & 0 & 1 & 0 \\
\hline 1.50 & 0.00 & 1.00 & 1 & 0 & 0 \\
\hline 0.91 & 0.00 & 3.50 & 1 & 1 & 0 \\
\hline 0.65 & 0.19 & 1.53 & 0 & 0 & 0 \\
\hline
\end{tabular}

Table 9 Ratios: Low-Energy Discharge.

\begin{tabular}{|c|c|c|c|c|c|}
\hline \multicolumn{3}{|c|}{ Ratio } & \multicolumn{3}{|c|}{ Ratio Group } \\
\hline R1 & R2 & R5 & R1 & R2 & R5 \\
\hline 0.26 & 2.15 & 1.18 & 0 & 1 & 1 \\
\hline 0.33 & 3.36 & 4.88 & 0 & 2 & 2 \\
\hline 0.17 & 18.54 & 8.67 & 0 & 2 & 2 \\
\hline 0.22 & 4.57 & 10.02 & 0 & 2 & 2 \\
\hline 0.13 & 2.97 & 8.63 & 0 & 1 & 2 \\
\hline 0.13 & 2.88 & 8.46 & 0 & 1 & 2 \\
\hline 0.17 & 1.00 & 1.00 & 0 & 1 & 1 \\
\hline 0.11 & 3.55 & inf & 0 & 2 & 2 \\
\hline 0.15 & 6.11 & 11.39 & 0 & 2 & 2 \\
\hline
\end{tabular}

Table 10 Ratios: High-Energy Discharge.

\begin{tabular}{|c|c|c|c|c|c|}
\hline \multicolumn{3}{|c|}{ Ratio } & \multicolumn{3}{c|}{ Ratio Group } \\
\hline R1 & R2 & R5 & R1 & R2 & R5 \\
\hline 0.20 & 2.49 & 16.00 & 0 & 1 & 2 \\
\hline 0.20 & 1.83 & 8.50 & 0 & 1 & 2 \\
\hline 0.39 & 1.85 & 14.40 & 0 & 1 & 2 \\
\hline 0.26 & 1.49 & inf & 0 & 1 & 2 \\
\hline 0.24 & 1.56 & 9.56 & 0 & 1 & 2 \\
\hline 0.20 & 1.45 & 12.47 & 0 & 1 & 2 \\
\hline 0.52 & 1.35 & 4.76 & 0 & 1 & 2 \\
\hline 0.26 & 1.42 & inf & 0 & 1 & 2 \\
\hline 0.30 & 1.14 & 12.63 & 0 & 1 & 2 \\
\hline
\end{tabular}

Table 11 Ratios: Thermal-Low.

\begin{tabular}{|c|c|c|c|c|c|}
\hline \multicolumn{3}{|c|}{ Ratio } & \multicolumn{3}{c|}{ Ratio Group } \\
\hline R1 & R2 & R5 & R1 & R2 & R5 \\
\hline 2.72 & 0.01 & 2.67 & 2 & 0 & 1 \\
\hline 2.30 & 0.00 & 4.66 & 2 & 0 & 2 \\
\hline 1.69 & 0.03 & 1.00 & 2 & 0 & 1 \\
\hline 27.00 & 0.25 & 0.08 & 2 & 1 & 0 \\
\hline 1.74 & 0.00 & 3.08 & 2 & 0 & 2 \\
\hline 12.71 & 0.00 & 0.34 & 2 & 0 & 0 \\
\hline 1.50 & 0.00 & 1.00 & 2 & 0 & 1 \\
\hline 0.91 & 0.00 & 3.50 & 0 & 0 & 2 \\
\hline 0.65 & 0.19 & 1.53 & 0 & 1 & 1 \\
\hline
\end{tabular}

Table 12 Ratios: Thermal-High.

\begin{tabular}{|c|c|c|c|c|c|}
\hline \multicolumn{3}{|c|}{ Ratio } & \multicolumn{3}{c|}{ Ratio Group } \\
\hline R1 & R2 & R5 & R1 & R2 & R5 \\
\hline 7.28 & 0.00 & 1.33 & 2 & 0 & 1 \\
\hline 1.57 & 0.04 & 12.64 & 2 & 0 & 2 \\
\hline 1.45 & 0.01 & 9.10 & 2 & 0 & 2 \\
\hline 3.33 & 0.03 & 6.05 & 2 & 0 & 2 \\
\hline 4.20 & 0.00 & 2.82 & 2 & 0 & 1 \\
\hline 2.68 & 0.15 & inf & 2 & 1 & 2 \\
\hline 1.94 & 0.02 & 68.33 & 2 & 0 & 2 \\
\hline 0.15 & 0.18 & 6.67 & 0 & 1 & 2 \\
\hline 2.35 & 0.03 & 3.90 & 2 & 0 & 2 \\
\hline
\end{tabular}

Ratio Groups are arranged into observation table as shown in Table 13 to Table 17 where:

- H_PD: Hypothesis Partial Discharge.

- H_LE: Hypothesis Low-Energy Discharge.

- H_HE: Hypothesis High-Energy Discharge.

- H_TL: Hypothesis Thermal-Low.

- H_TH: Hypothesis Thermal-High. 
K. O. Bachri et al. / Advances in Science, Technology and Engineering Systems Journal Vol. 2, No. 3, 1137-1146 (2017)

Table 13 Observation: Partial Discharge.

\begin{tabular}{|c|c|c|c|c|c|c|c|}
\hline \multirow{2}{*}{$\begin{array}{l}\mathrm{N}^{\text {th }} \\
\text { Obs. }\end{array}$} & \multirow{2}{*}{ sensors } & \multirow{2}{*}{$\begin{array}{l}\text { Range } \\
\text { Group }\end{array}$} & \multicolumn{5}{|c|}{ hypotheses } \\
\hline & & & H_PD & H_LE & H_HE & H_TL & H_TH \\
\hline \multirow{3}{*}{1} & R1 & 1 & 1 & 1 & 1 & 0 & 0 \\
\hline & R2 & 2 & 0 & 0 & 0 & 0 & 1 \\
\hline & R5 & 0 & 1 & 0 & 0 & 0 & 0 \\
\hline \multirow{3}{*}{2} & R1 & 1 & 1 & 1 & 1 & 0 & 0 \\
\hline & R2 & 1 & 1 & 0 & 0 & 0 & 0 \\
\hline & R5 & 0 & 1 & 0 & 0 & 0 & 0 \\
\hline \multirow{3}{*}{3} & R1 & 0 & 0 & 0 & 0 & 1 & 1 \\
\hline & R2 & 2 & 0 & 0 & 0 & 0 & 1 \\
\hline & R5 & 2 & 0 & 0 & 1 & 0 & 1 \\
\hline \multirow{3}{*}{4} & R1 & 0 & 0 & 0 & 0 & 1 & 1 \\
\hline & R2 & 2 & 0 & 0 & 0 & 0 & 1 \\
\hline & R5 & 0 & 1 & 0 & 0 & 0 & 0 \\
\hline \multirow{3}{*}{5} & R1 & 0 & 0 & 0 & 0 & 1 & 1 \\
\hline & R2 & 1 & 1 & 0 & 0 & 0 & 0 \\
\hline & R5 & 0 & 1 & 0 & 0 & 0 & 0 \\
\hline \multirow{3}{*}{6} & R1 & 0 & 0 & 0 & 0 & 1 & 1 \\
\hline & R2 & 1 & 1 & 0 & 0 & 0 & 0 \\
\hline & R5 & 0 & 1 & 0 & 0 & 0 & 0 \\
\hline \multirow{3}{*}{7} & R1 & 1 & 1 & 1 & 1 & 0 & 0 \\
\hline & R2 & 0 & 0 & 1 & 1 & 1 & 0 \\
\hline & R5 & 0 & 1 & 0 & 0 & 0 & 0 \\
\hline \multirow{3}{*}{8} & R1 & 1 & 1 & 1 & 1 & 0 & 0 \\
\hline & R2 & 1 & 1 & 0 & 0 & 0 & 0 \\
\hline & R5 & 0 & 1 & 0 & 0 & 0 & 0 \\
\hline \multirow{3}{*}{9} & R1 & 0 & 0 & 0 & 0 & 1 & 1 \\
\hline & R2 & 0 & 0 & 1 & 1 & 1 & 0 \\
\hline & R5 & 0 & 1 & 0 & 0 & 0 & 0 \\
\hline
\end{tabular}

Table 14 Observation: Low-Energy Discharge.

\begin{tabular}{|c|c|c|c|c|c|c|c|}
\hline \multirow{2}{*}{$\begin{array}{l}\mathrm{N}^{\text {th }} \\
\text { Obs. }\end{array}$} & \multirow{2}{*}{ sensors } & \multirow{2}{*}{$\begin{array}{l}\text { Range } \\
\text { Group }\end{array}$} & \multicolumn{5}{|c|}{ hypotheses } \\
\hline & & & H_PD & H_LE & H_HE & H_TL & H_TH \\
\hline \multirow{3}{*}{1} & R1 & 0 & 0 & 1 & 1 & 1 & 1 \\
\hline & R2 & 1 & 1 & 1 & 1 & 0 & 0 \\
\hline & R5 & 1 & 0 & 1 & 0 & 1 & 0 \\
\hline \multirow{3}{*}{2} & R1 & 0 & 0 & 1 & 1 & 1 & 1 \\
\hline & R2 & 2 & 0 & 1 & 0 & 0 & 1 \\
\hline & R5 & 2 & 0 & 1 & 1 & 0 & 1 \\
\hline \multirow{3}{*}{3} & R1 & 0 & 0 & 1 & 1 & 1 & 1 \\
\hline & R2 & 2 & 0 & 1 & 0 & 0 & 1 \\
\hline & R5 & 2 & 0 & 1 & 1 & 0 & 1 \\
\hline \multirow{3}{*}{4} & R1 & 0 & 0 & 1 & 1 & 1 & 1 \\
\hline & R2 & 2 & 0 & 1 & 0 & 0 & 1 \\
\hline & R5 & 2 & 0 & 1 & 1 & 0 & 1 \\
\hline
\end{tabular}

\begin{tabular}{|l|l|l|l|l|l|l|l|}
\hline \multirow{4}{*}{5} & $\mathrm{R} 1$ & 0 & 0 & 1 & 1 & 1 & 1 \\
\cline { 2 - 8 } & $\mathrm{R} 2$ & 1 & 1 & 1 & 1 & 0 & 0 \\
\cline { 2 - 8 } & $\mathrm{R} 5$ & 2 & 0 & 1 & 1 & 0 & 1 \\
\hline \multirow{4}{*}{6} & $\mathrm{R} 1$ & 0 & 0 & 1 & 1 & 1 & 1 \\
\cline { 2 - 8 } & $\mathrm{R} 2$ & 1 & 1 & 1 & 1 & 0 & 0 \\
\cline { 2 - 8 } & $\mathrm{R} 5$ & 2 & 0 & 1 & 1 & 0 & 1 \\
\hline \multirow{4}{*}{8} & $\mathrm{R} 1$ & 0 & 0 & 1 & 1 & 1 & 1 \\
\cline { 2 - 8 } & $\mathrm{R} 2$ & 1 & 1 & 1 & 1 & 0 & 0 \\
\cline { 2 - 8 } & $\mathrm{R} 5$ & 1 & 0 & 1 & 0 & 1 & 0 \\
\hline \multirow{4}{*}{8} & $\mathrm{R} 1$ & 0 & 0 & 1 & 1 & 1 & 1 \\
\cline { 2 - 8 } & $\mathrm{R} 2$ & 2 & 0 & 1 & 0 & 0 & 1 \\
\cline { 2 - 8 } & $\mathrm{R} 5$ & 2 & 0 & 1 & 1 & 0 & 1 \\
\hline \multirow{3}{*}{9} & $\mathrm{R} 1$ & 0 & 0 & 1 & 1 & 1 & 1 \\
\cline { 2 - 8 } & $\mathrm{R} 2$ & 2 & 0 & 1 & 0 & 0 & 1 \\
\cline { 2 - 8 } & $\mathrm{R} 5$ & 2 & 0 & 1 & 1 & 0 & 1 \\
\hline
\end{tabular}

Table 15 Observation: High-Energy Discharge.

\begin{tabular}{|c|c|c|c|c|c|c|c|}
\hline \multirow{2}{*}{$\begin{array}{l}\mathrm{N}^{\text {th }} \\
\text { Obs. }\end{array}$} & \multirow{2}{*}{ sensors } & \multirow{2}{*}{$\begin{array}{l}\text { Range } \\
\text { Group }\end{array}$} & \multicolumn{5}{|c|}{ hypotheses } \\
\hline & & & H_PD & H_LE & H_HE & H_TL & H_TH \\
\hline \multirow{3}{*}{1} & R1 & 0 & 0 & 1 & 1 & 1 & 1 \\
\hline & R2 & 1 & 1 & 1 & 1 & 0 & 0 \\
\hline & R5 & 2 & 0 & 1 & 1 & 0 & 1 \\
\hline \multirow{3}{*}{2} & R1 & 0 & 0 & 1 & 1 & 1 & 1 \\
\hline & R2 & 1 & 1 & 1 & 1 & 0 & 0 \\
\hline & R5 & 2 & 0 & 1 & 1 & 0 & 1 \\
\hline \multirow{3}{*}{3} & R1 & 0 & 0 & 1 & 1 & 1 & 1 \\
\hline & R2 & 1 & 1 & 1 & 1 & 0 & 0 \\
\hline & R5 & 2 & 0 & 1 & 1 & 0 & 1 \\
\hline \multirow{3}{*}{4} & R1 & 0 & 0 & 1 & 1 & 1 & 1 \\
\hline & R2 & 1 & 1 & 1 & 1 & 0 & 0 \\
\hline & R5 & 2 & 0 & 1 & 1 & 0 & 1 \\
\hline \multirow{3}{*}{5} & $\mathrm{R} 1$ & 0 & 0 & 1 & 1 & 1 & 1 \\
\hline & R2 & 1 & 1 & 1 & 1 & 0 & 0 \\
\hline & R5 & 2 & 0 & 1 & 1 & 0 & 1 \\
\hline \multirow{3}{*}{6} & R1 & 0 & 0 & 1 & 1 & 1 & 1 \\
\hline & R2 & 1 & 1 & 1 & 1 & 0 & 0 \\
\hline & R5 & 2 & 0 & 1 & 1 & 0 & 1 \\
\hline \multirow{3}{*}{7} & R1 & 0 & 0 & 1 & 1 & 1 & 1 \\
\hline & R2 & 1 & 1 & 1 & 1 & 0 & 0 \\
\hline & R5 & 2 & 0 & 1 & 1 & 0 & 1 \\
\hline \multirow{3}{*}{8} & $\mathrm{R} 1$ & 0 & 0 & 1 & 1 & 1 & 1 \\
\hline & R2 & 1 & 1 & 1 & 1 & 0 & 0 \\
\hline & $\mathrm{s}$ & 2 & 0 & 1 & 1 & 0 & 1 \\
\hline \multirow{3}{*}{9} & $\mathrm{R} 1$ & 0 & 0 & 1 & 1 & 1 & 1 \\
\hline & $\mathrm{R} 2$ & 1 & 1 & 1 & 1 & 0 & 0 \\
\hline & R5 & 2 & 0 & 1 & 1 & 0 & 1 \\
\hline
\end{tabular}


K. O. Bachri et al. / Advances in Science, Technology and Engineering Systems Journal Vol. 2, No. 3, 1137-1146 (2017)

Table 16 Observation: Thermal-Low.

\begin{tabular}{|c|c|c|c|c|c|c|c|}
\hline \multirow{2}{*}{$\begin{array}{l}\mathrm{N}^{\text {th }} \\
\text { Obs. }\end{array}$} & \multirow{2}{*}{ sensors } & \multirow{2}{*}{$\begin{array}{l}\text { Range } \\
\text { Group }\end{array}$} & \multicolumn{5}{|c|}{ hypotheses } \\
\hline & & & H_PD & H_LE & H_HE & H_TL & H_TH \\
\hline \multirow{3}{*}{1} & $\mathrm{R} 1$ & 2 & 0 & 0 & 0 & 1 & 1 \\
\hline & $\mathrm{R} 2$ & 0 & 0 & 0 & 0 & 1 & 0 \\
\hline & R5 & 1 & 1 & 1 & 0 & 1 & 0 \\
\hline \multirow{3}{*}{2} & R1 & 2 & 0 & 0 & 0 & 1 & 0 \\
\hline & R2 & 0 & 0 & 0 & 0 & 1 & 0 \\
\hline & R5 & 2 & 0 & 1 & 1 & 0 & 1 \\
\hline \multirow{3}{*}{3} & $\mathrm{R} 1$ & 2 & 0 & 0 & 0 & 1 & 0 \\
\hline & $\mathrm{R} 2$ & 0 & 0 & 0 & 0 & 1 & 0 \\
\hline & R5 & 1 & 1 & 1 & 0 & 1 & 0 \\
\hline \multirow{3}{*}{4} & R1 & 2 & 0 & 0 & 0 & 1 & 0 \\
\hline & $\mathrm{R} 2$ & 1 & 1 & 1 & 1 & 0 & 0 \\
\hline & R5 & 0 & 0 & 0 & 0 & 1 & 0 \\
\hline \multirow{3}{*}{5} & $\mathrm{R} 1$ & 2 & 0 & 0 & 0 & 1 & 0 \\
\hline & $\mathrm{R} 2$ & 0 & 0 & 0 & 0 & 1 & 0 \\
\hline & R5 & 2 & 0 & 1 & 1 & 0 & 1 \\
\hline \multirow{3}{*}{6} & $\mathrm{R} 1$ & 2 & 0 & 0 & 0 & 1 & 0 \\
\hline & $\mathrm{R} 2$ & 0 & 0 & 0 & 0 & 1 & 0 \\
\hline & R5 & 0 & 0 & 0 & 0 & 1 & 0 \\
\hline \multirow{3}{*}{7} & $\mathrm{R} 1$ & 2 & 0 & 0 & 0 & 1 & 0 \\
\hline & $\mathrm{R} 2$ & 0 & 0 & 0 & 0 & 1 & 0 \\
\hline & R5 & 1 & 1 & 1 & 0 & 1 & 0 \\
\hline \multirow{3}{*}{8} & $\mathrm{R} 1$ & 0 & 0 & 1 & 1 & 1 & 1 \\
\hline & $\mathrm{R} 2$ & 0 & 0 & 0 & 0 & 1 & 0 \\
\hline & R5 & 2 & 0 & 1 & 1 & 0 & 1 \\
\hline \multirow{3}{*}{9} & $\mathrm{R} 1$ & 0 & 0 & 1 & 1 & 1 & 1 \\
\hline & $\mathrm{R} 2$ & 1 & 1 & 1 & 1 & 0 & 0 \\
\hline & R5 & 1 & 1 & 1 & 0 & 1 & 0 \\
\hline
\end{tabular}

Table 17 Observation: Thermal-High.

\begin{tabular}{|c|c|c|c|c|c|c|c|}
\hline \multirow{2}{*}{$\begin{array}{l}\mathrm{N}^{\text {th }} \\
\text { Obs. }\end{array}$} & \multirow{2}{*}{ sensors } & \multirow{2}{*}{ quantity } & \multicolumn{5}{|c|}{ hypotheses } \\
\hline & & & H_PD & H_LE & H_HE & H_TL & H_TH \\
\hline \multirow{3}{*}{1} & R1 & 2 & 0 & 0 & 0 & 1 & 1 \\
\hline & $\mathrm{R} 2$ & 0 & 0 & 0 & 0 & 1 & 1 \\
\hline & R5 & 1 & 1 & 1 & 0 & 1 & 1 \\
\hline \multirow{3}{*}{2} & $\mathrm{R} 1$ & 2 & 0 & 0 & 0 & 1 & 1 \\
\hline & R2 & 0 & 0 & 0 & 0 & 1 & 1 \\
\hline & R5 & 2 & 0 & 1 & 1 & 0 & 1 \\
\hline \multirow{3}{*}{3} & R1 & 2 & 0 & 0 & 0 & 1 & 1 \\
\hline & R2 & 0 & 0 & 0 & 0 & 1 & 1 \\
\hline & R5 & 2 & 0 & 1 & 1 & 0 & 1 \\
\hline \multirow{3}{*}{4} & $\mathrm{R} 1$ & 2 & 0 & 0 & 0 & 1 & 1 \\
\hline & $\mathrm{R} 2$ & 0 & 0 & 0 & 0 & 1 & 1 \\
\hline & R5 & 2 & 0 & 1 & 1 & 0 & 1 \\
\hline
\end{tabular}

\begin{tabular}{|c|c|c|c|c|c|c|c|}
\hline \multirow{4}{*}{5} & R1 & 2 & 0 & 0 & 0 & 1 & 1 \\
\cline { 2 - 8 } & R2 & 0 & 0 & 0 & 0 & 1 & 1 \\
\cline { 2 - 8 } & R5 & 1 & 1 & 1 & 0 & 1 & 1 \\
\hline \multirow{4}{*}{6} & R1 & 2 & 0 & 0 & 0 & 1 & 1 \\
\cline { 2 - 8 } & R2 & 1 & 1 & 1 & 1 & 0 & 0 \\
\cline { 2 - 8 } & R5 & 2 & 0 & 1 & 1 & 0 & 1 \\
\hline \multirow{4}{*}{7} & R1 & 2 & 0 & 0 & 0 & 1 & 1 \\
\cline { 2 - 9 } & R2 & 0 & 0 & 0 & 0 & 1 & 1 \\
\cline { 2 - 9 } 8 & R5 & 2 & 0 & 1 & 1 & 0 & 1 \\
\hline \multirow{4}{*}{8} & R1 & 0 & 0 & 1 & 1 & 1 & 0 \\
\cline { 2 - 8 } & R2 & 1 & 1 & 1 & 1 & 0 & 0 \\
\cline { 2 - 8 } & R5 & 2 & 0 & 1 & 1 & 0 & 1 \\
\hline \multirow{3}{*}{9} & R1 & 2 & 0 & 0 & 0 & 1 & 1 \\
\cline { 2 - 8 } & R2 & 0 & 0 & 0 & 0 & 1 & 1 \\
\cline { 2 - 8 } & R5 & 2 & 0 & 1 & 1 & 0 & 1 \\
\hline
\end{tabular}

New Knowledge Probability Distribution (NKPD) is shown in Table 18 to Table 22.

Table 18 NKPD: Partial Discharge.

\begin{tabular}{|c|c|c|c|c|}
\hline H_PD & H_LE & H_HE & H_TL & H_TH \\
\hline 0.44 & 0.11 & 0.11 & 0.00 & 0.33 \\
\hline 0.78 & 0.11 & 0.11 & 0.00 & 0.00 \\
\hline 0.00 & 0.00 & 0.17 & 0.17 & 0.67 \\
\hline 0.33 & 0.00 & 0.00 & 0.17 & 0.50 \\
\hline 0.67 & 0.00 & 0.00 & 0.17 & 0.17 \\
\hline 0.67 & 0.00 & 0.00 & 0.17 & 0.17 \\
\hline 0.44 & 0.22 & 0.22 & 0.11 & 0.00 \\
\hline 0.78 & 0.11 & 0.11 & 0.00 & 0.00 \\
\hline 0.33 & 0.11 & 0.11 & 0.28 & 0.17 \\
\hline
\end{tabular}

Table 18 shows hypothesis H_PD has the highest value of Degree of Certainty (DoC) on seven out of nine samples, while the other two do not provide an accurate interpretation.

Table 19 NKPD: Low-Energy Discharge.

\begin{tabular}{|c|c|c|c|c|}
\hline H_PD & H_LE & H_HE & H_TL & H_TH \\
\hline 0.11 & 0.36 & 0.19 & 0.25 & 0.08 \\
\hline 0.00 & 0.36 & 0.19 & 0.08 & 0.36 \\
\hline 0.00 & 0.36 & 0.19 & 0.08 & 0.36 \\
\hline 0.00 & 0.36 & 0.19 & 0.08 & 0.36 \\
\hline 0.11 & 0.31 & 0.31 & 0.08 & 0.19 \\
\hline 0.11 & 0.31 & 0.31 & 0.08 & 0.19 \\
\hline 0.11 & 0.36 & 0.19 & 0.25 & 0.08 \\
\hline 0.00 & 0.36 & 0.19 & 0.08 & 0.36 \\
\hline 0.00 & 0.36 & 0.19 & 0.08 & 0.36 \\
\hline
\end{tabular}


K. O. Bachri et al. / Advances in Science, Technology and Engineering Systems Journal Vol. 2, No. 3, 1137-1146 (2017)

Table 19 shows hypothesis H_LE has the highest value of Degree of Certainty (DoC) on nine out of nine samples with most of them showing other hypothesis with significant DoC.

Table 20 NKPD: High-Energy Discharge.

\begin{tabular}{|c|c|c|c|c|}
\hline H_PD & H_LE & H_HE & H_TL & H_TH \\
\hline 0.11 & 0.31 & 0.31 & 0.08 & 0.19 \\
\hline 0.11 & 0.31 & 0.31 & 0.08 & 0.19 \\
\hline 0.11 & 0.31 & 0.31 & 0.08 & 0.19 \\
\hline 0.11 & 0.31 & 0.31 & 0.08 & 0.19 \\
\hline 0.11 & 0.31 & 0.31 & 0.08 & 0.19 \\
\hline 0.11 & 0.31 & 0.31 & 0.08 & 0.19 \\
\hline 0.11 & 0.31 & 0.31 & 0.08 & 0.19 \\
\hline 0.11 & 0.31 & 0.31 & 0.08 & 0.19 \\
\hline 0.11 & 0.31 & 0.31 & 0.08 & 0.19 \\
\hline
\end{tabular}

Table 20 shows hypothesis H_HE has the highest value of Degree of Certainty (DoC) on nine out of nine samples with all of them showing significant H_LE and other hypothesis with less significant DoC.

Table 21 NKPD: Thermal-Low.

\begin{tabular}{|c|c|c|c|c|}
\hline H_PD & H_LE & H_HE & H_TL & H_TH \\
\hline 0.11 & 0.11 & 0.00 & 0.61 & 0.17 \\
\hline 0.00 & 0.11 & 0.11 & 0.67 & 0.11 \\
\hline 0.11 & 0.11 & 0.00 & 0.78 & 0.00 \\
\hline 0.11 & 0.11 & 0.11 & 0.67 & 0.00 \\
\hline 0.00 & 0.11 & 0.11 & 0.67 & 0.11 \\
\hline 0.00 & 0.00 & 0.00 & 1.00 & 0.00 \\
\hline 0.11 & 0.11 & 0.00 & 0.78 & 0.00 \\
\hline 0.00 & 0.19 & 0.19 & 0.42 & 0.19 \\
\hline 0.22 & 0.31 & 0.19 & 0.19 & 0.08 \\
\hline
\end{tabular}

Table 21 shows hypothesis H_TL has the highest value of Degree of Certainty (DoC) on eight out of nine samples with some of them showing other hypotheses with less significant DoC.

Table 22 NKPD: Thermal-High.

\begin{tabular}{|c|c|c|c|c|}
\hline H_PD & H_LE & H_HE & H_TL & H_TH \\
\hline 0.08 & 0.08 & 0.00 & 0.42 & 0.42 \\
\hline 0.00 & 0.11 & 0.11 & 0.33 & 0.44 \\
\hline 0.00 & 0.11 & 0.11 & 0.33 & 0.44 \\
\hline 0.00 & 0.11 & 0.11 & 0.33 & 0.44 \\
\hline 0.08 & 0.08 & 0.00 & 0.42 & 0.42 \\
\hline 0.11 & 0.22 & 0.22 & 0.17 & 0.28 \\
\hline 0.00 & 0.11 & 0.11 & 0.33 & 0.44 \\
\hline 0.11 & 0.33 & 0.33 & 0.11 & 0.11 \\
\hline 0.00 & 0.11 & 0.11 & 0.33 & 0.44 \\
\hline
\end{tabular}

Table 22 shows hypothesis H_TH has the highest value of Degree of Certainty (DoC) on eight out of nine samples with all of them showing other hypotheses with significant DoC.

The overall accuracy of A3S algorithm is $91.1 \%$ as shown in Table 23.

Table 23 NKPD: Thermal-High.

\begin{tabular}{|c|c|}
\hline Fault Identification & Accuracy (\%) \\
\hline Partial Discharge & 77.8 \\
\hline Low-Energy Discharge & 100 \\
\hline High-Energy Discharge & 100 \\
\hline Thermal Low & 88.9 \\
\hline Thermal-High & 88.9 \\
\hline Average & $\mathbf{9 1 . 1}$ \\
\hline
\end{tabular}

The algorithm is then used to calculate transformer condition and make interpretation of the calculated condition based on DGA data. The condition of a transformer depends on several factors. In this research, the condition is calculated is based on DGA data. Table 24 shows Dissolved Gas Analysis (DGA) data collected from PLN and Table 25 shows other quantities included in transformer condition factors.

Table 24 DGA data

\begin{tabular}{|r|r|r|r|r|r|r|}
\cline { 2 - 8 } \multicolumn{1}{c|}{} & \multicolumn{6}{c|}{ gas concentration (ppm) } \\
\hline $\mathrm{t}$ (days) & \multicolumn{1}{c|}{$\mathrm{H}_{2}$} & \multicolumn{1}{c|}{$\mathrm{CH}_{4}$} & $\mathrm{C}_{2} \mathrm{H}_{6}$ & $\mathrm{C}_{2} \mathrm{H}_{4}$ & $\mathrm{C}_{2} \mathrm{H}_{2}$ & \multicolumn{1}{c|}{$\mathrm{CO}$} \\
\hline 0 & 20.00 & 94.54 & 62.87 & 0.00 & 0.00 & 27.87 \\
\hline 486 & 20.00 & 0.00 & 80.97 & 15.54 & 0.00 & 6.58 \\
\hline 551 & 58.01 & 120.32 & 169.23 & 3.26 & 0.00 & 205.42 \\
\hline 586 & 439.47 & 137.21 & 121.72 & 63.06 & 57.12 & 0.00 \\
\hline 873 & 380.04 & 137.39 & 122.89 & 71.24 & 75.69 & 135.97 \\
\hline 884 & 315.90 & 156.73 & 123.19 & 56.56 & 79.04 & 55.53 \\
\hline 961 & 276.17 & 172.71 & 114.88 & 74.86 & 70.50 & 171.51 \\
\hline 985 & 48.24 & 94.83 & 83.03 & 47.84 & 29.93 & 0.00 \\
\hline 990 & 165.21 & 156.14 & 163.52 & 72.09 & 40.07 & 120.90 \\
\hline 1011 & 42.91 & 152.12 & 157.53 & 58.60 & 36.10 & 226.49 \\
\hline 1374 & 39.84 & 36.84 & 199.27 & 60.37 & 14.07 & 35.55 \\
\hline 1692 & 20.00 & 172.92 & 208.30 & 51.90 & 6.63 & 305.14 \\
\hline 1882 & 20.00 & 162.52 & 179.81 & 34.24 & 0.00 & 0.00 \\
\hline 2034 & 321.12 & 146.04 & 210.90 & 35.13 & 0.00 & 516.79 \\
\hline 2203 & 20.00 & 70.33 & 236.11 & 10.74 & 0.00 & 0.00 \\
\hline 2315 & 20.00 & 26.75 & 87.71 & 10.93 & 0.00 & 0.00 \\
\hline 2316 & 20.00 & 48.67 & 75.74 & 10.58 & 0.00 & 100.40 \\
\hline
\end{tabular}


K. O. Bachri et al. / Advances in Science, Technology and Engineering Systems Journal Vol. 2, No. 3, 1137-1146 (2017)

Table 25 Dielectric, water content, and acid number data

\begin{tabular}{|c|c|c|c|}
\hline $\begin{array}{c}\text { Dielectric } \\
\text { Breakdown } \\
(\mathrm{kV} / 2.5 \mathrm{~mm})\end{array}$ & $\begin{array}{l}\text { Water } \\
\text { Content } \\
\text { (ppm) }\end{array}$ & $\begin{array}{c}\text { IFT } \\
(\text { dyne } / \mathrm{cm})\end{array}$ & $\begin{array}{c}\text { Acid } \\
\text { Number } \\
(\mathrm{mg} \mathrm{KOH} / \mathrm{g})\end{array}$ \\
\hline- & 5.96 & 41.00 & 0.08 \\
\hline- & 5.96 & 30.00 & 0.10 \\
\hline- & 5.96 & 29.00 & 0.14 \\
\hline- & 5.96 & 31.00 & 0.17 \\
\hline- & 5.96 & 30.00 & 0.24 \\
\hline- & 5.96 & 30.00 & 0.23 \\
\hline- & 5.96 & 31.00 & 0.14 \\
\hline- & 5.96 & 31.00 & 0.15 \\
\hline- & 5.96 & 32.00 & 0.15 \\
\hline- & 5.96 & 32.00 & 0.22 \\
\hline 68.40 & 5.96 & 32.00 & 0.20 \\
\hline 66.00 & 5.96 & 34.20 & 0.14 \\
\hline 50.20 & 5.96 & 33.80 & 0.13 \\
\hline 52.60 & 4.13 & 33.40 & 0.13 \\
\hline 50.10 & 4.13 & 32.20 & 0.11 \\
\hline 55.20 & 4.13 & 32.90 & 0.11 \\
\hline 43.10 & 4.13 & 32.60 & 0.11 \\
\hline
\end{tabular}

Data are compared to standards $[7,8]$ and are given scores. The results are shown in Table 26 and Table 27.

Table 26 Condition grouping and scoring based on DGA

\begin{tabular}{|r|c|c|c|c|c|c|}
\cline { 2 - 7 } \multicolumn{1}{c|}{} & \multicolumn{6}{c|}{ Condition Scoring Based on DGA } \\
\hline $\begin{array}{c}\mathrm{t} \\
\text { (days) }\end{array}$ & $\mathrm{H}_{2}$ & $\mathrm{CH}_{4}$ & $\mathrm{C}_{2} \mathrm{H}_{6}$ & $\mathrm{C}_{2} \mathrm{H}_{4}$ & $\mathrm{C}_{2} \mathrm{H}_{2}$ & $\mathrm{CO}$ \\
\hline 0 & 100 & 100 & 100 & 100 & 100 & 100 \\
\hline 486 & 100 & 100 & 67 & 100 & 100 & 100 \\
\hline 551 & 100 & 67 & 0 & 100 & 100 & 100 \\
\hline 586 & 67 & 67 & 33 & 67 & 0 & 100 \\
\hline 873 & 67 & 67 & 33 & 67 & 0 & 100 \\
\hline 884 & 67 & 67 & 33 & 67 & 0 & 100 \\
\hline 961 & 67 & 67 & 33 & 67 & 0 & 100 \\
\hline 985 & 100 & 100 & 67 & 100 & 33 & 100 \\
\hline 990 & 67 & 67 & 0 & 67 & 0 & 100 \\
\hline 1011 & 100 & 67 & 0 & 67 & 0 & 100 \\
\hline 1374 & 100 & 100 & 0 & 67 & 33 & 100 \\
\hline 1692 & 100 & 67 & 0 & 67 & 67 & 100 \\
\hline 1882 & 100 & 67 & 0 & 100 & 100 & 100 \\
\hline 2034 & 67 & 67 & 0 & 100 & 100 & 67 \\
\hline 2203 & 100 & 100 & 0 & 100 & 100 & 100 \\
\hline 2315 & 100 & 100 & 67 & 100 & 100 & 100 \\
\hline
\end{tabular}

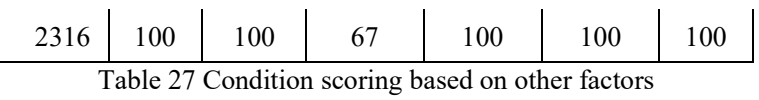

\begin{tabular}{|c|c|c|c|c|}
\cline { 2 - 5 } \multicolumn{1}{c|}{} & \multicolumn{4}{c}{ Condition Scoring Based on Other Factors } \\
\hline $\mathrm{t}$ (days) & $\begin{array}{c}\text { Dielectric } \\
\text { Strength }\end{array}$ & $\begin{array}{c}\text { Water } \\
\text { Content }\end{array}$ & IFT & $\begin{array}{c}\text { Acid } \\
\text { Number }\end{array}$ \\
\hline 0 & - & 100 & 100 & 50 \\
\hline 486 & - & 100 & 0 & 50 \\
\hline 551 & - & 100 & 0 & 50 \\
\hline 586 & - & 100 & 33 & 0 \\
\hline 873 & - & 100 & 0 & 0 \\
\hline 884 & - & 100 & 0 & 0 \\
\hline 961 & - & 100 & 33 & 50 \\
\hline 985 & - & 100 & 33 & 0 \\
\hline 990 & - & 100 & 33 & 0 \\
\hline 1011 & - & 100 & 33 & 0 \\
\hline 1374 & - & 100 & 33 & 0 \\
\hline 1692 & 100 & 100 & 33 & 50 \\
\hline 1882 & 100 & 100 & 33 & 50 \\
\hline 2034 & 75 & 100 & 33 & 50 \\
\hline 2203 & 75 & 100 & 33 & 50 \\
\hline 2315 & 75 & 100 & 33 & 50 \\
\hline 2316 & 75 & 100 & 33 & 50 \\
\hline
\end{tabular}

The total condition of the transformer is shown in Table 28 .

Table 28 Transformer total condition

\begin{tabular}{|c|c|}
\hline $\begin{array}{c}\mathrm{t} \\
\text { (days) }\end{array}$ & $\begin{array}{c}\text { total } \\
\text { condition }\end{array}$ \\
\hline 0 & 94 \\
\hline 486 & 80 \\
\hline 551 & 69 \\
\hline 586 & 52 \\
\hline 873 & 48 \\
\hline 884 & 48 \\
\hline 961 & 57 \\
\hline 985 & 70 \\
\hline 990 & 48 \\
\hline 1011 & 52 \\
\hline 1374 & 59 \\
\hline 1692 & 68 \\
\hline 1882 & 75 \\
\hline 2034 & 66 \\
\hline 2203 & 77 \\
\hline 2315 & 84 \\
\hline 2316 & 84 \\
\hline
\end{tabular}


K. O. Bachri et al. / Advances in Science, Technology and Engineering Systems Journal Vol. 2, No. 3, 1137-1146 (2017)

The total condition can be drawn in form of Figure 5.

\begin{tabular}{|l|l|l|l|l|l|l|}
2316 & 85 & 0.0000 & 1 & 0 & 1 & 1 \\
\hline
\end{tabular}

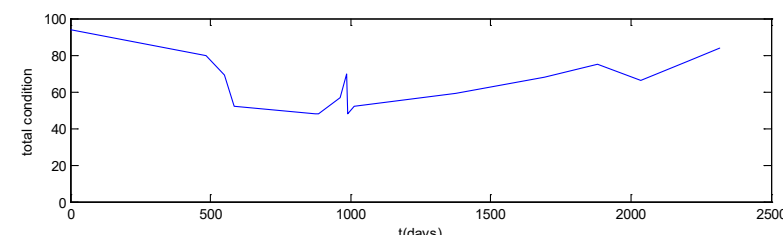

Figure 5 Condition curve

At first, transformer condition has high value, as time passes, it decreases due to the degradation process. The degradation process produces gases, acid, water, which accelerate the degradation process.

There is a sudden increase in condition and then followed by a sudden decrease, this is probably caused by the maintenance process and the setting process after maintenance. After maintenance, the condition began to increase with small gradient.

The gradient of condition is shown in Figure 6.

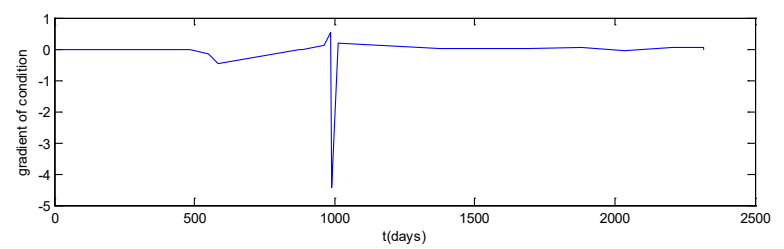

Figure 6 Gradient of condition

The gradient of condition depends on condition. In general, it is relatively stable. There is a sudden decrease at day 990 and then followed by sudden increase.

The observation table is shown in Table 29.

Table 29 Observation table

\begin{tabular}{|r|c|c|c|c|c|c|}
\hline \multirow{2}{*}{$\begin{array}{c}\mathrm{t} \\
\text { (days) }\end{array}$} & \multirow{2}{*}{ cond } & \multirow{2}{*}{ gradient } & \multirow{2}{*}{ SC } & SG & \multicolumn{2}{|c|}{ NKPD } \\
\hline 0 & 94 & 0.0000 & 1 & 0 & 1 & 1 \\
\hline 486 & 80 & -0.0305 & 1 & 0 & 1 & 1 \\
\hline 551 & 69 & -0.1709 & 1 & 1 & 0 & 1 \\
\hline 586 & 52 & -0.4762 & 1 & 1 & 1 & 0 \\
\hline 873 & 48 & -0.0129 & 0 & 0 & 1 & 1 \\
\hline 884 & 48 & 0.0000 & 0 & 0 & 1 & 1 \\
\hline 961 & 57 & 0.1203 & 1 & 1 & 1 & 0 \\
\hline 985 & 70 & 0.5401 & 1 & 1 & 0 & 1 \\
\hline 990 & 48 & -4.4444 & 0 & 1 & 1 & 0 \\
\hline 1011 & 52 & 0.1764 & 1 & 1 & 1 & 0 \\
\hline 1374 & 59 & 0.0204 & 1 & 0 & 1 & 1 \\
\hline 1692 & 68 & 0.0277 & 1 & 0 & 1 & 1 \\
\hline 1882 & 76 & 0.0390 & 1 & 0 & 1 & 1 \\
\hline 2034 & 66 & -0.0624 & 1 & 1 & 1 & 1 \\
\hline 2203 & 77 & 0.0657 & 1 & 1 & 1 & 1 \\
\hline 2315 & 85 & 0.0661 & 1 & 1 & 1 & 1 \\
\hline & & & & & & \\
\hline 0 & & & & & & 1 \\
\hline
\end{tabular}

NKPD is knowledge of the system at each time of observation. To obtain knowledge growth, NKPD is fused with the previous NKPD, in this case, NKPD from the beginning of observation. This process produced NKPD over time (NKPDT)

Knowledge growth is represented by NKPDT can be calculated using (6) and is shown in Table 30. Knowledge growth can also be represented using Figure 7 .

Table 30 NKPDT

\begin{tabular}{|c|c|c|}
\hline \multirow{4}{*}{$\begin{array}{c}\mathrm{t} \\
\text { (days) }\end{array}$} & \multicolumn{2}{|c|}{$\mathrm{NKPD}$} \\
\cline { 2 - 4 } & $\mathrm{HW}$ & $\mathrm{HL}$ \\
\hline 0 & 0.5000 & 0.5000 \\
\hline 486 & 0.5000 & 0.5000 \\
\hline 551 & 0.3333 & 0.6667 \\
\hline 586 & 0.2500 & 0.7500 \\
\hline 873 & 0.3000 & 0.7000 \\
\hline 884 & 0.3333 & 0.6667 \\
\hline 961 & 0.2857 & 0.7143 \\
\hline 985 & 0.2500 & 0.7500 \\
\hline 990 & 0.3333 & 0.6667 \\
\hline 1011 & 0.3000 & 0.7000 \\
\hline 1374 & 0.3182 & 0.6818 \\
\hline 1692 & 0.3333 & 0.6667 \\
\hline 1882 & 0.3462 & 0.6538 \\
\hline 2034 & 0.3214 & 0.6786 \\
\hline 2203 & 0.3000 & 0.7000 \\
\hline 2315 & 0.2813 & 0.7188 \\
\hline 2316 & 0.2941 & 0.7059 \\
\hline & &
\end{tabular}

Where:

- Cond: condition of the transformer

- Gradient: condition change over time

- SC: cond, after compared to standard

- SG: gradient, after compared to standard 


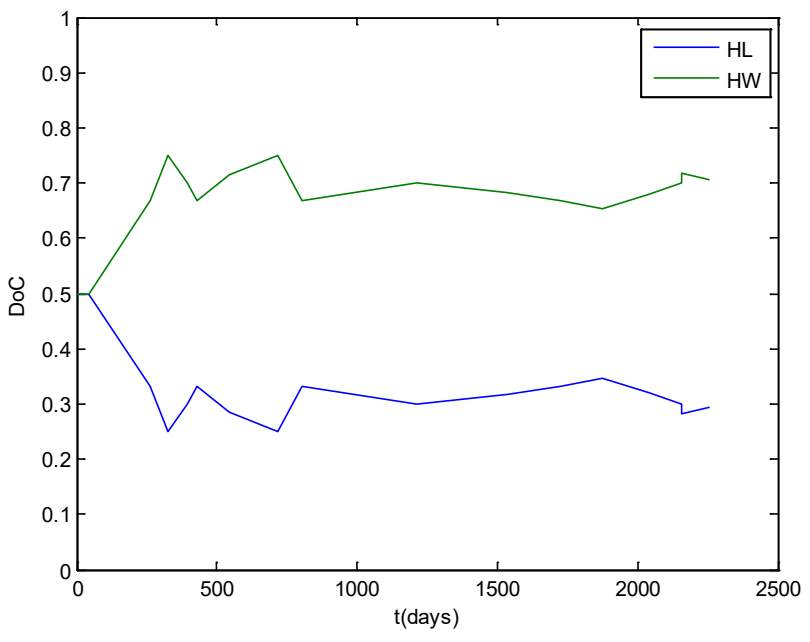

Figure 7 Hypotheses/Knowledge growth

Where HL shows Hypothesis-Life and HW shows Hypothesis-Warning. Both hypotheses show the same value at the first observation. As time passes, HW increases, while HL decreases. It indicates that the transformer condition is in the limit and there are some changes in the gradient. The positive gradient shows maintenance, while negative condition shows degradation.

Spikes in Figure 7 shows there is an occurrence of a phenomenon indicating a hypothesis. In this case, there is a change in gradient of condition, making it in a warning condition. As gradient is dependent to condition, the hypothesis HW is dependent to HL as well.

\section{Concluding Remarks}

A3S has successfully interpreted DGA data to identified fault based on the classified dataset. It has successfully identified not only the main fault, which has the most significant DoC. It has successfully identified the fault(s) occurred along with the main fault, which has less significant of DoC. This feature acts as the early warning system.

OMA3S has successfully interpreted transformer condition by fusing the parameter Condition (SC) and Gradient (SG) to produce Hypothesis-Life (HL) and Hypothesis-Warning (HW). HL and HW both are fused with the previous values to obtain knowledge growth.

In the next research, more parameters will be included and elaborated to increase accuracy. The number of hypothesis will be added as well to reduce the direct impact of the change of one hypothesis to the other when using only two hypotheses.

This algorithm will be implemented in form of a processor called cognitive processor. Using a special purposes processor will have advantages, such as energy efficiency and minimize disturbance caused by electromagnetic transmission.

\section{Conflict of Interest}

Hereby, The authors would like to declares that this article "Cognitive Artificial Intelligence Method for Interpreting Transformer Condition Based on Maintenance Data" is an original work of our research group and has no conflict of interests.

\section{Acknowledgment}

The first author would like to appreciate Yokeu Wibisana for the maintenance data and Harry Gumilang of PLN for the discussion and information provided.

\section{References}

[1] A. S. Ahmad and K. O. Bachri, "Cognitive artificial intelligence method for measuring transformer performance," 2016 Future Technologies Conference (FTC), San Francisco, CA, 2016, pp. 67-73.

[2] L. Po-Chun and G. Jyh-Cherng,"'Research on Transforming Condition-Based Maintenance System using the Method of Fuzzy Comprehensive Evaluation", World Academy of Science, Engineering and Technology, vol. 5, 2011

[3] H. Gumilang, "Hydrolysis process in PLN P3BJB transformers as an effect of oil insulation oxidation," 2012 IEEE International Conference on Condition Monitoring and Diagnosis, Bali, 2012, pp. 1147-1150.

[4] A. D. W. Sumari, A. S. Ahmad, A. I. Wuryandari, J. Sembiring, "Brain Inspired Knowledge Growing System: Towards a True Cognitive Agent", IJCSAI Vol. 2 Issue 1, 2012

[5] K. O. Bachri, B. Anggoro, A. S. Ahmad, "Transformer Performance Calculation Using Information Fusion based on DGA Interpretation”, 2016 IEEE International Symposium on Electronics and Smart Devices (ISESD), Lembang, 2016.

[6] Z. Qi, W. Zhongdong, and P. A. CROSSLEY, "Power Transformer End-oflife Modeling: Linking Statistical Approach with Physical Ageing Process", CIRED Workshop, June 2010.

[7] IEEE Guide for the Interpretation of Gases Generated in Oil-Immersed Transformers," in IEEE Std C57.104-2008 (Revision of IEEE Std C57.1041991), vol., no., pp.1-36, Feb. 22009

[8] IEEE Guide for Acceptance and Maintenance of Insulating Oil in Equipment," in IEEE Std C57.106-2006 (Revision of IEEE Std C57.1062002), vol., no., pp.1-36, Dec. 62006.

[9] M. Duvall, A. dePablo,"'nterpretation of Gas-In-Oil Analysis Using New IEC Publication 60599 and IEC TC 10 Databases" IEEE Insulation Magazine, Vol. 17, No. 2, March/April 2001, Feature Article. 